\title{
Measuring online volitional response control with a continuous tracking task
}

\author{
SHARON MOREIN-ZAMIR, ROMEO CHUA, IAN FRANKS, \\ PAUL NAGELKERKE, and ALAN KINGSTONE \\ University of British Columbia, Vancouver, British Columbia, Canada
}

\begin{abstract}
We present a new tracking task designed to measure elements of response control in particular types of response adjustments such as stopping. In this task, participants track a visual target by manually pressing on a force sensor to yield a trace of force over time. Hardware specifications are detailed, as is an algorithm for determining the latencies of response adjustments such as stopping. We illustrate the use of the task in two experiments. Experiment 1 explores the reliability of data produced by the task. Experiment 2 examines some of the issues that can be addressed using the new task. These results demonstrate the usefulness and potential of the task for gauging response control within the context of the stopping literature.
\end{abstract}

Numerous tasks have been employed to measure cognitive influences on motor control, including error correction and the adjustment of an ongoing response such as accelerating, decelerating, or changing direction of a movement (see, e.g., Henry \& Harrison, 1961; Megaw, 1972; Vince \& Welford, 1967; for a review, see Logan \& Cowan, 1984). One of the most notable measures has been that of response inhibition, or stopping (Lappin \& Eriksen, 1966; Logan, 1994). The advantage of studying stopping, over other forms of control, has been that it is a clear and extreme form of control that is easy to observe and relatively easy to measure (Logan, 1994). Stopping has traditionally been studied using the stop signal task, in which participants are asked to press a key in response to a "go signal," which indicates that a response is to be executed. Occasionally the go signal is followed by a "stop signal," which indicates that the keypress is to be stopped and the intended action withheld. Although no overt behavior is observed on a successful stop trial, the use of a racehorse model, together with the task, allows for the mathematical estimation of the latency of stopping, or stop signal reaction time (SSRT; Logan \& Cowan, 1984). SSRT serves as a direct measure of the control exerted to prevent the execution of a volitional goal-directed action. To date, the stop signal task has been used in a wide variety of settings in order to examine questions ranging from the ballistic elements of movement to the inhibitory underpinnings of disorders such as attention deficit hyperactivity disorder (ADHD; De Jong, Coles, Logan, \& Gratton, 1990; Nigg, 2001; Oosterlaan, Logan, \& Sergeant, 1998; Osman, Kornblum, \& Meyer, 1986).

This research was supported by grants to A.K. from NSERC, the Michael Smith Foundation of Health Research, and the Human Early Learning Partnership, and to I.F. and R.C. from NSERC. Correspondence concerning this article should be addressed to S. Morein-Zamir, Department of Psychiatry, University of Cambridge, Box 189, Addenbrooke's Hospital CB2 2QQ, England (e-mail: sm658@ac.uk).
The purpose of the present study is to introduce and explore an alternative task for measuring stopping, which offers several advantages over the traditional stop signal task (Morein-Zamir \& Meiran, 2003). For instance, the task allows for the exploration of additional instances of control, such as response adjustment and stopping after response execution, which have been largely neglected with the increasing popularity of the stop signal task. In addition, the new task is not dependent on a mathematical model such as the racehorse model or its assumptions, as is the stop signal task, and so is resistant to instances in which the model may not hold (see, e.g., Logan \& Burkell, 1986). Likewise, the alternative task can provide a measurement of stopping RT on each trial, which is not possible with the stop signal task, providing for more efficient data collection and a broader range of analyses. Specifically, questions that require measurements on every trial can be addressed, including the examination of sequential effects and measurements of variance. Such measures are important because they allow for the testability of predictions that to date could not be confirmed (see, e.g., Band, van der Molen, \& Logan, 2003; Tannock, 2003).

The standard version of the tracking task requires participants to stop an ongoing action after it has been initiated (for a similar rationale, see Ladefoged, Silverstein, \& Papcun, 1973, and Logan, 1982). On each trial, participants engage in tracking a moving visual target presented on the screen, and at some later point, they respond to a signal to stop their action. Stopping performance is measured by examining the response adjustment onset latency to the stop signal (Henry \& Harrison, 1961). In the present task, the target moves along a circular trajectory at a constant angular speed, and participants control the speed of a corresponding response marker by pressing on a force sensor (the greater the pressing force, the faster the response marker rotation). Participants perform the tracking task for a variable amount of time before a stop signal is presented. Onset of response adjustment - that is, force 
reduction causing rotational deceleration — is taken as the measure of control.

The present study introduces the new task and its development in detail. Data collected over two experiments explore the performance characteristics of the task. In the second experiment, onset of increasing force leading to marker acceleration is introduced as an additional measure of response adjustment, and is compared with onset of stopping.

\section{GENERAL METHOD}

\section{Apparatus}

Although the development of the tracking task has been a dynamic process, the core logic and motivation defining the task and the dependent measures have remained constant. The hardware on which the task was implemented has changed over the course of experiments conducted. The initial development was performed with participants pressing on a $7.6 \times 10.2 \times .9 \mathrm{~cm}$ custom-machined aluminum load cell instrumented with four strain gauges (Omega model $350 \Omega$ ). Additional data were collected with responses executed using a telegraph key instrumented with two strain gauges to measure downward force applied to the finger rest. In both cases, the analog signals from the strain gauges were amplified (Northwood Instruments, model IA-102-500). The resulting force-voltage signal was filtered (Krone-Hite 3750 analog filter set at $50 \mathrm{~Hz}$ low pass) and then sampled at the $\pm 1.25 \mathrm{~V}$ range at $1000 \mathrm{~Hz}$ by an analog-todigital (A/D) converter (Techmar Labmaster) installed in the datacollection computer. This computer (with an Intel Pentium I, 233$\mathrm{MHz}$ processor and $16 \mathrm{MB}$ RAM) was running Microsoft Windows 98 Second Edition in DOS mode, which controlled stimulus presentation and data collection via custom computer software (written in Borland Turbo Pascal 6.0). Stimuli were presented to participants on a Zenith 14-in. flat screen monitor (ZCM-1490) with a refresh rate of $60 \mathrm{~Hz}$ running in standard VGA video mode (resolution $640 \times 480$ pixels), and the experimenter monitored performance on a monochrome monitor with a Hercules-compatible monochrome video card. Additional data collection was performed with participants' response force measured on the selector button of a modified older model Macintosh computer mouse. The computer mouse was modified to accept a force sensor (Flexiforce A201-1) under the selector key. The sensor used a pressure-sensitive ink that changes its electrical resistance with compressive force; the resulting analog voltage was sampled by an A/D converter (Pico ADC-212) at $1000 \mathrm{~Hz}$. This apparatus was connected to the parallel port of a 320CDT Toshiba laptop, which controlled stimulus display and response collection. The same display was presented on the laptop monitor as well as a slaved monitor, with performance summary data being presented at the end of each practice trial and at the end of each experimental block. In all cases, performance data were stored and analyzed after data collection.

\section{Procedure}

In the development of the new task, many different parameters and their specific values had to be defined. To ensure a satisfactory task, several pilot versions were developed and tested. In some of the experiments, several of the parameters were changed or manipulated and the task was further modified. The following is a description of the initial task, also detailing how particular parameters were chosen.

Initially, we determined several parameters after inspecting one of the earlier response inhibition tasks, employed by Slater-Hammel (1960). Additional guidelines were adopted from a tracking task using a similar rationale (Morein-Zamir \& Meiran, 2003). In this prior task, participants performed a visual tracking task, but the apparatus and measurement of dependent measures differed considerably. With the aid of a computer mouse, the participants tracked a visual target moving in a series of linear trajectories. Deceleration onset latency, or SSRT, was calculated using an accumulative regression algorithm calculated from the spatial location of the mouse marker over time. In this task, although stopping could be measured on each trial, motor noise and a poor sampling rate $(50 \mathrm{~Hz})$ caused the algorithm to fail on a substantial portion of the trials $(22 \%$ and $33 \%$ of trials in Experiments 1 and 2, respectively).

The present task possessed several important new characteristics. The task was more straightforward and the tracking requirements were constrained. Participants controlled the rotational speed of the response marker but not its direction, thus restricting the noise resulting from motor performance. In addition, the dependent measure was computed from the force applied over time. This force controlled the response marker with an increased temporal resolution of $1000 \mathrm{~Hz}$. The main dependent measure was conceptually similar to SSRT in the previous tracking task and consisted of the latency to initiate a stop - that is, to initiate a reduction in applied force on the sensor, causing angular deceleration of the tracking response. However, rather than computing SSRT from the position of the computer mouse and hence from the position of the response marker on the screen, we now computed SSRT from the force-over-time profiles, allowing for increased reliability. This alternate dependent measure enabled the measurement of SSRT on almost every trial.

The presented version of the task proved to be challenging and engaging, yet after a few minutes of practice, performance was stable for the vast majority of participants. This proved to be an important guideline in determining task characteristics. Trials were considered stable if, during the SSRT latency interval, the tracking force did not deviate more than $100 \mathrm{~g}$ and the maximum tracking error was less than $15^{\circ}$. Consequently, performance yielded force profiles that were sufficiently steady so as to allow a clear demarcation of SSRT on over $98 \%$ of all trials.

In the present tracking task, on each trial, the target marker moved in a predetermined way, rotating around the screen at a fixed speed (see Figure 1). Both target and response images were small circular markers. The participants were asked to track and advance the response marker so as to overlap the target marker. To do this, the participants pressed on a static force sensor to advance their response marker. The more force was applied, the faster the response marker rotated. On a typical stop trial, after a variable period of tracking, a signal appeared, indicating that participants were to stop tracking. They did this by terminating the applied pressure on the force sensor without moving their finger; as a consequence, the response marker also stopped moving. We stressed to the participants that stopping was defined as releasing the exerted force, without lifting the finger from the key, to ensure maximal similarity between conceptual and motor stopping (MoreinZamir, Nagelkerke, Chua, Franks, \& Kingstone, 2004). SSRT was determined to be the point in time at which the applied force on the sensor began a drop toward the initial baseline prepress force. When tracking was stable, with no large changes in tracking force, this point was quite distinct, as can be seen in Figure 2.

The stimulus and response markers first appeared stationary at the 3 o'clock position and then rotated clockwise around a circular path with a $200-$ pixel $(8.1-\mathrm{cm})$ radius. The size of this pathway was constrained by the height of the screen. Circular stimuli were chosen because they do not have a directional component and thus would appear the same, no matter where along the pathway they were presented. Several colors of the circles were tested, including a red target and a green response marker, but bright blue and green were eventually selected because they have little meaning in combination. The sizes of the stimuli were chosen so that the response marker fit inside the target during perfect tracking performance. A radius of 5-7 pixels for the green response circle and a radius of 8-10 pixels for the blue target ring were chosen. This entailed a radius of $0.3 \mathrm{~cm}$ for the green response circle and $0.3-0.405 \mathrm{~cm}$ for the blue target ring. The size was sufficiently large to allow for easy visibility but was small enough to require participants to fixate on a particular area of the screen. Different rotational speeds of the target were also examined. A stimulus rotation rate of $1 \mathrm{~Hz}$ was tested and 
deemed too fast for adequate tracking performance, so a rotation rate of $0.5 \mathrm{~Hz}$ was chosen.

A vertical downward force of $3.924 \mathrm{~N}$, equivalent to a $400 \mathrm{-g}$ mass resting on the sensor, gave a response rotation rate of $0.5 \mathrm{~Hz}$, matching the stimulus rotation speed. There was a linear force-rotation rate relationship of $0.125 \mathrm{~Hz}$ for every $0.981 \mathrm{~N}$ (the force exerted by a $100-\mathrm{g}$ mass at 1 gravity). The equipment was calibrated using standard gram masses, and the units of force are reported in equivalent gram mass units, for simplicity. The minimal threshold of $100 \mathrm{~g}$ $(0.981 \mathrm{~N})$ yielded a $0.125-\mathrm{Hz}$ rate, and the maximum threshold of $2,000 \mathrm{~g}(19.62 \mathrm{~N})$ resulted in a response rotation rate of $2.5 \mathrm{~Hz}$. The optimal tracking force was reduced from an initial $600 \mathrm{~g}$ to $400 \mathrm{~g}$, to reduce participant fatigue. At $400 \mathrm{~g}$, the force was sustainable for durations up to $1 \mathrm{~h}$, and yet the apparatus was not overly sensitive.

\section{Analysis of Dependent Measures}

A response usually consisted of a time interval with zero applied force on the selector key, followed by a rapid onset peaking at $600 \mathrm{~g}$ or more (often 1,000 g, applied to "catch up" with the target), followed by tracking at the target value of $400 \mathrm{~g}$ (see Figure 2). After the presentation of a stop signal, there was a marked force release, whereby tracking force decreased from approximately $400 \mathrm{~g}$ back to $0 \mathrm{~g}$. If an acceleration signal was introduced instead of a stop signal (see below), participants increased the applied force well above $400 \mathrm{~g}$ for several seconds. The dependent measures were SSRT and ACCEL RT for the onset of force reduction and force increase leading to stopping and accelerating, respectively. In both cases, performance adjustment RTs were measured as the latency from signal onset to the onset of the response adjustment.

The onset and offset points for changes in applied force were found by analyzing the change in the response force profile over the time course of the trial (Figure 2). First, the force profile for each trial was digitally filtered at $10 \mathrm{~Hz}$. A standard procedure (see below) was used to detect an adjustment on the force profile. Spe- cifically, the procedure could be used to find any of three possible points: (1) following a stop signal, force reduction latency leading to deceleration; (2) following an acceleration signal, force increase latency leading to acceleration; and (3) following target movement at the beginning of a trial, the force onset latency initiating the tracking movement. ${ }^{1} \mathrm{~A}$ similar procedure could also be used to examine the latency at which the force decrease or increase terminated. In the case of a force increase leading to acceleration, this value was not reliable, because it was heavily dependent on the final speed of the response marker following force increase, which was under the discretion of the participant and varied across trials. Thus, response adjustment onset and not offset times were the main dependent measures. Nevertheless, the algorithm for finding force change offset is also described below.

To find the onset of force reduction, a computer algorithm searched for the first point following the stop signal at which the measured force dropped below $200 \mathrm{~g}$ (midway between the ideal $400 \mathrm{~g}$ going to $0 \mathrm{~g}$ ). The rate of change, or slope, of this point was calculated based on the value difference of the two points immediately surrounding it divided by the time interval between the points. The algorithm then searched for the first preceding data point at which the slope was $10 \%$ of this value. This point in time was marked as the onset of force reduction, and the latency was calculated as the difference between this point in time and the signal onset time marker. The algorithm also worked forward in time from the 200-g threshold to find the point at which the slope was $10 \%$ of this value. The difference between this time and the target signal provided the latency at which the applied force reduction and consequent marker deceleration was essentially complete (also called final RT or FINRT). The calculation of onset latency for a force increase was almost identical. The onset of a force increase was determined on the basis of the point whose slope was $10 \%$ of the slope measured at the $600 \mathrm{~g}$ threshold. The PASCAL code for the core algorithm is shown in the Appendix.

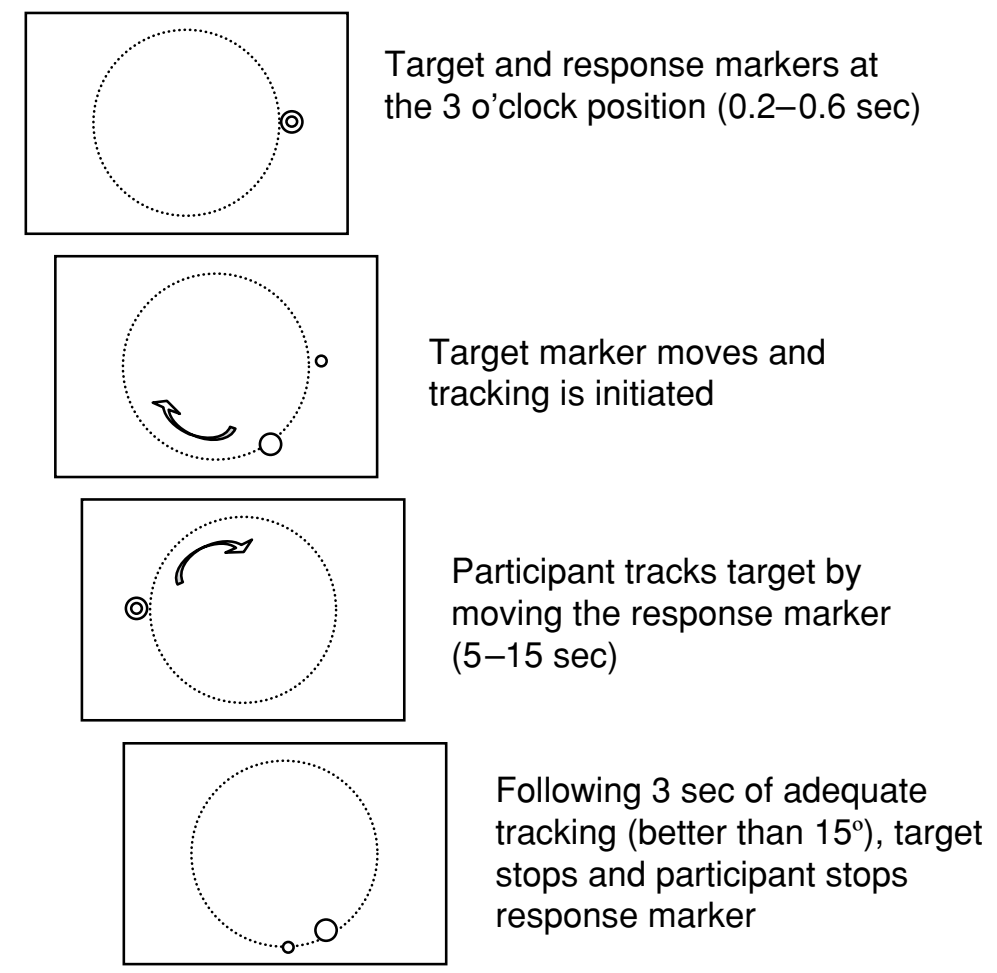

Figure 1.A depiction of the typical series of events in the tracking task. 

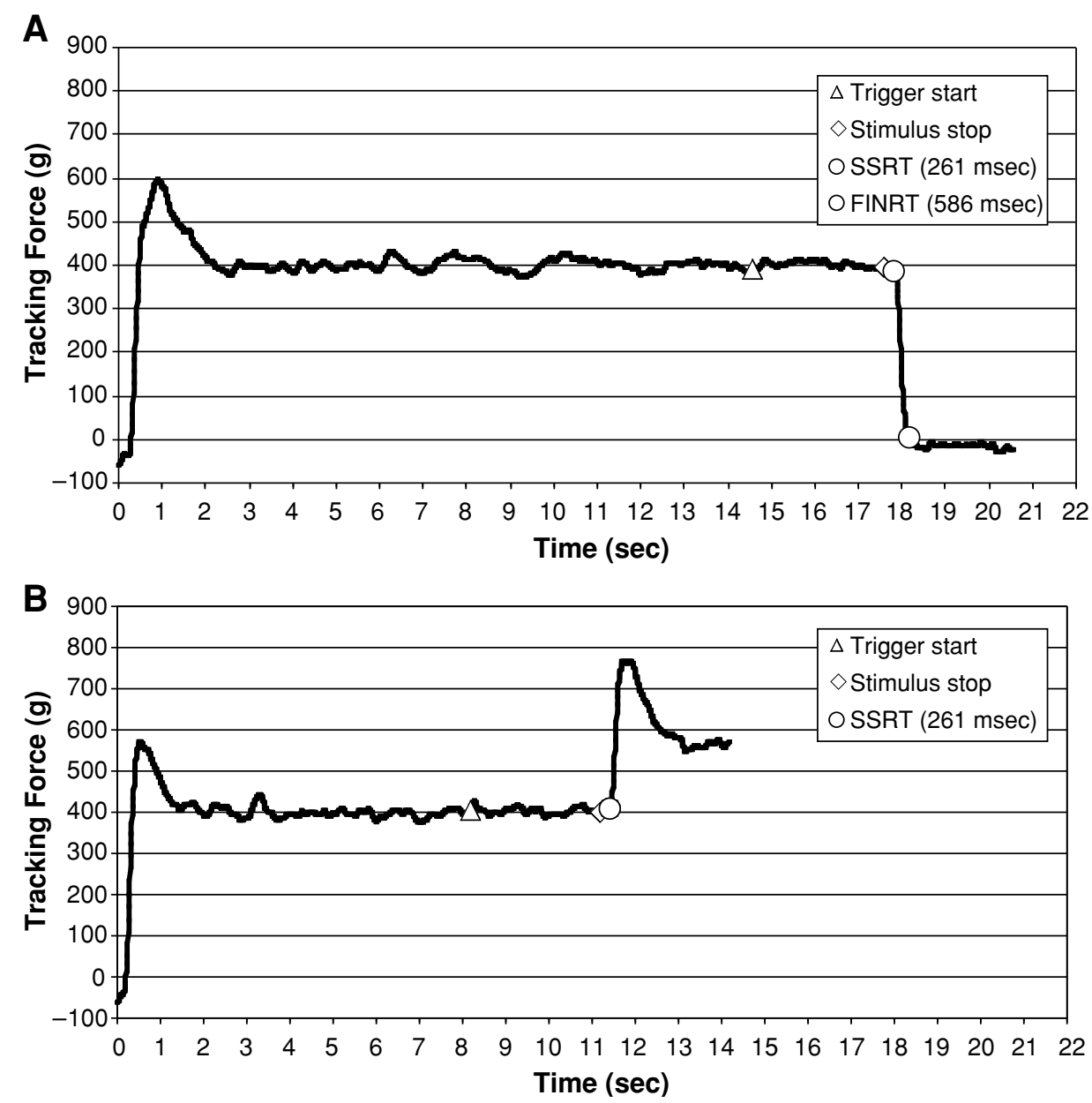

Figure 2. An example of the force-over-time profile yielded over the course of a trial. Figure 2A portrays a stop trial, whereas Figure 2B portrays an accelerate trial (see Experiment 2 for further details of Figure 2B).

In essence, the algorithm was used to calculate the force differential of the trial. The slope for each data point was calculated as the difference between the values before and the value after, divided by the time interval, yielding the change force in units of $\mathrm{g} / \mathrm{sec}$. This resulted in a positive value when force increased and a negative value when force decreased. Peak onset slopes typically measured $1,000 \mathrm{~g} / \mathrm{sec}$. This algorithm was applied to all trials to find force onset and offset, and was followed by visual inspection of every trial. We specifically screened trials for SSRT and FINRT times, and tracked stability as indicated by the force variability during the SSRT interval (between signal onset and force change onset). Only trials with stable tracking data during the SSRT interval with clear force change onsets and offsets were included for data analysis. Because the final version of the task led to very stable tracking at the moment of stimulus presentation, less than $2 \%$ of trials were excluded (see the Results sections). Trials with SSRT values larger than 3.5 standard deviations were also excluded from further analyses. Finally, SSRT latencies shorter than $50 \mathrm{msec}$ were considered anticipations and also were not included in the analyses.

An additional measure of interest was tracking performance on each trial. This was measured by phase error (PE). PE was calculated as the absolute angular position error between the stimulus and response markers displayed on the computer screen along the circular trajectory, and ranged from $0^{\circ}$ to $180^{\circ}$. Thus, a smaller value connoted better performance. The PE value on each trial consti- tuted the average angle for $1 \mathrm{sec}$ before signal onset, sampled at $1000 \mathrm{~Hz}$.

\section{Exploratory Data Collection}

In the following section, we present some initial results generated using the tracking task. The following two experiments and their corresponding data allowed for the initial exploration and verification of the task.

\section{EXPERIMENT 1}

The purpose of the first experiment was to test the new task and obtain a body of data for which descriptive statistics could be obtained and verified. The data analysis algorithm was tested and examined on a sample of observations. This experiment also illustrates the typical performance on the task and the types of descriptive statistics that can be gathered.

\section{Method}

Participants. Ten undergraduates from the Psychology Department at the University of British Columbia participated in exchange for course credit (mean age was 19.3 years, $S D=0.7$ ). All 
but one were right-handed, two were male, and all had normal or corrected-to-normal vision. After being informed about the nature of the experiments, the participants signed an informed consent form in accordance with the ethical guidelines of the University of British Columbia.

Procedure and Design. Using the methods described above, the participants completed one session of approximately 100 experimental trials (see Table 1, below), with a stop signal on each trial. The trials began with stationary target and response stimuli at the 3 o'clock position of the imaginary circle. After a random delay of 200-600 msec, the target marker began circling clockwise at $0.5 \mathrm{~Hz}$. After a random tracking duration of 5-15 sec, the program "armed" the stop signal. Following this arming time, the program monitored tracking performance so that when the PE was less than $15^{\circ}$ for $3,000 \mathrm{msec}$, the stop signal was triggered. The stop signal was the stopping of the target stimulus, indicating that participants were to stop as quickly as possible. The intertrial duration was $2 \mathrm{sec}$. After 10 practice trials, the experimental trials began, with selfterminating breaks every 20 trials.

\section{Results and Discussion}

Using the results of Experiment 1, we developed the analysis algorithm, and over $99 \%$ of the trials yielded valid SSRT values. Trials on which SSRT could not be computed reliably, or that resulted in values larger than $590 \mathrm{msec}$ or smaller than $50 \mathrm{msec}(0.9 \%)$, were subsequently dropped from all analyses. Table 1 provides descriptive statistics for SSRT, FINRT, and PE for each participant. SSRT values were in close agreement with those that have previously been found in the stop signal procedure (see, e.g., Logan, 1994; Logan \& Cowan, 1984) and those found in the mouse-tracking task (Morein-Zamir \& Meiran, 2003). The shape of the SSRT distribution across participants proved to be similar to that of most RT functions. The box- and whiskerplot graphs (see Figure 3) support this conclusion. Some variability was observed between participants, with Participant 4 being slower overall to stop, and Participant 9 being best overall in the tracking task.

Correlations between SSRT, final RT, and PE across participants indicated that SSRT and FINRT were significantly correlated $[t(8)=2.7, r=.69, p<.05]$. Although both SSRT and FINRT correlated negatively with PE, neither correlation reached significance in the present sample
( -.32 and -.47 , respectively). Correlations were further computed between each of the three measures within each participant (see Table 2). This analysis explored whether participants were likely to trade off tracking and stopping performance. Mean correlations were computed on transformed Fisher $Z$ values and then retransformed (Rosenthal, 1991). The resulting mean correlation within each participant between SSRT and PE was .01, and between FINRT and $P E$ it was .03, suggesting that even on individual trials, there was no correlation between tracking and stopping performance (see Morein-Zamir \& Meiran, 2003, for a similar conclusion). There was a strong, positive correlation of .74 between SSRT and FINRT. Hence, PE does not seem to correlate consistently with either of the stopping measures, although they correlate significantly between themselves. Correlations between each of the dependent measures and trial number were also computed, using the above procedures, to examine the effects of practice across time in the experimental trials. Practice had no effects across trials, with all correlations being below 08 .

Finally, the stability of the main measure of SSRT was examined. Specifically, we questioned whether the number of trials used to measure mean SSRT would alter the resulting values. Therefore, mean SSRT was computed for the first 15, 20, 30, 40, 50, 60, and 80 trials and compared with mean SSRT computed on all trials. When the measures were entered into an ANOVA, we observed no significant differences between them $(F<1)$. All mean SSRT measures with 20 observations and above were within $4 \mathrm{msec}$ of each other $(302 \mathrm{msec})$. Mean SSRTs of the first 10 and 15 observations were $8 \mathrm{msec}$ slower. Correlations between SSRT measurements for the first 20 observations and above ranged from .94 to .99 and were highly significant. These analyses suggest that 20 observations are sufficient to gauge the mean SSRT value.

In conclusion, the first study provided descriptive data indicating that the present task could indeed be used to gain measurement of the initial stopping times. In accordance with Morein-Zamir and Meiran (2003), the primary measurements were SSRT and PE. Since FINRT correlated highly with SSRT, but was also sensitive to tracking

Table 1

Number of Trials, Means, Standard Deviations, and Medians for SSRT, FINRT, and PE for Each Participant in Experiment 1

\begin{tabular}{|c|c|c|c|c|c|c|c|c|c|c|}
\hline \multirow{2}{*}{$\begin{array}{c}\text { P } \\
\text { No. }\end{array}$} & \multirow{2}{*}{$\begin{array}{l}\text { No. of } \\
\text { Trials }\end{array}$} & \multicolumn{3}{|c|}{ SSRT } & \multicolumn{3}{|c|}{ FINRT } & \multicolumn{3}{|c|}{ PE } \\
\hline & & $M$ & $S D$ & $M d n$ & $M$ & $S D$ & $M d n$ & $M$ & $S D$ & $M d n$ \\
\hline 1 & 59 & 300 & 56 & 292 & 483 & 69 & 468.0 & 4.0 & 1.9 & 4.0 \\
\hline 2 & 119 & 257 & 49 & 254 & 389 & 47 & 379.0 & 3.8 & 2.2 & 3.7 \\
\hline 3 & 100 & 312 & 51 & 307 & 540 & 86 & 540.5 & 2.9 & 1.8 & 2.8 \\
\hline 4 & 98 & 356 & 54 & 358 & 633 & 60 & 631.5 & 2.6 & 1.8 & 2.5 \\
\hline 5 & 98 & 279 & 42 & 278 & 598 & 79 & 586.5 & 4.1 & 2.4 & 3.9 \\
\hline 6 & 98 & 308 & 61 & 299 & 555 & 78 & 547.0 & 2.4 & 1.9 & 1.9 \\
\hline 7 & 120 & 287 & 36 & 291 & 538 & 58 & 539.5 & 2.5 & 2.1 & 2.1 \\
\hline 8 & 100 & 266 & 44 & 260 & 489 & 64 & 484 & 3.1 & 1.3 & 3.1 \\
\hline 9 & 100 & 291 & 56 & 288 & 612 & 114 & 617.5 & 1.9 & 1.3 & 1.6 \\
\hline \multirow[t]{2}{*}{10} & 94 & 338 & 60 & 334 & 604 & 72 & 594.0 & 3.1 & 2.0 & 2.7 \\
\hline & 99 & 299 & 50 & 295 & 544 & 72 & 539.0 & 3.0 & 1.9 & 2.77 \\
\hline
\end{tabular}

Note-P No., participant number; SSRT, stop signal RT; FINRT, final RT; PE, phase error; $M$, mean; $S D$, standard deviation; $M d n$, median. 
performance (as indicated by the higher correlation with $\mathrm{PE})$, it was deemed a redundant dependent measure. In following experiments, when possible, FINRT was calculated. However, it did not reveal any additional information beyond SSRT, and was often a more variable measure leading to less powerful analyses. Accordingly, FINRT is not reported in the following study.

\section{EXPERIMENT 2}

The second experiment sought to illustrate the types of research questions that can be investigated, and further explored the task and the resulting data. In the previous experiment, no manipulations were conducted. Thus, the present study set out to examine how performance on the task would appear when several conditions were introduced and conventional analyses (e.g., ANOVAs) were required. Likewise, we examined the reliability of the dependent measure algorithm. The first manipulation involved the type of response required. In one task, as in the previous experiment, the participants were instructed to stop as quickly as possible when observing the signal. In a second task, rather than stopping, the participants were asked to adjust their performance so as to increase their speed in response to the signal. Thus, participants increased the force as quickly as possible and accelerated the velocity of the response marker for a few seconds. The second manipulation involved the type of signal used. The first signal replicated the signal used in the previous experiment, that of the target stopping. The second signal was the changing of the target color while the target continued to rotate. It was hypothesized that the target stopping would be a more effective signal, because previous work has found that target location change resulted in faster adjustments of a pointing response than did target color change (Pisella, Arzi, \& Rossetti, 1998). On the basis of compatibility theory (Hommel \& Prinz, 1997; Proctor \& Reeve, 1990), it was also hypothesized that the target-stopping signal would be highly compatible with the stopping response. Correspondingly, an interaction was predicted in which the target-stopping signal would be more effective than the color-change signal in the stop task in comparison with the accelerate task.

\section{Method}

Participants. Twelve undergraduates from the Psychology Department at the University of British Columbia participated in exchange for course credit (mean age was 19.2 years, $S D=0.9$ ). All were right-handed, three were male, and all had normal or corrected-to-normal vision. After being informed about the nature of the experiments, the participants signed an informed consent form in accordance with the ethical guidelines of the University of British Columbia.

Procedure and Design. The present methods were similar to those used in Experiment 1, with the following exceptions. Two types of signals were employed in each task. The first stop signal was the same as in Experiment 1 and involved the target stimulus stopping. The second stop signal was a color change; the target marker continued to rotate. To ensure sufficient salience, the target color changed to white. The stop signals were randomly intermixed within each block of trials. In addition, there were now two separate tasks. The first task replicated the previous study, requiring that participants stop their tracking performance. The second task required that participants increase their applied force on the key in response to the signal, thus accelerating the response marker. The participants maintained the response marker at a faster rotational speed for several seconds, or for a sufficient time to circle the screen twice. Response marker acceleration onset latency, ACCEL RT, was defined as the time between stimulus onset and the onset of applied force increase. This was conceptually similar to SSRT, which was based on the onset of force decrease (see the Analysis section, above). The order of the two tasks was counterbalanced across participants. After 10 practice trials, each participant completed 100 experimental trials, 50 in each of the tasks. Self-terminating breaks were made available after the 17th and 34th trials in each block.

\section{Results and Discussion}

In keeping with the conclusions of the previous experiment, SSRT/ACCEL RT and PE were the main dependent measures. Trials on which SSRT/ACCEL RT could not

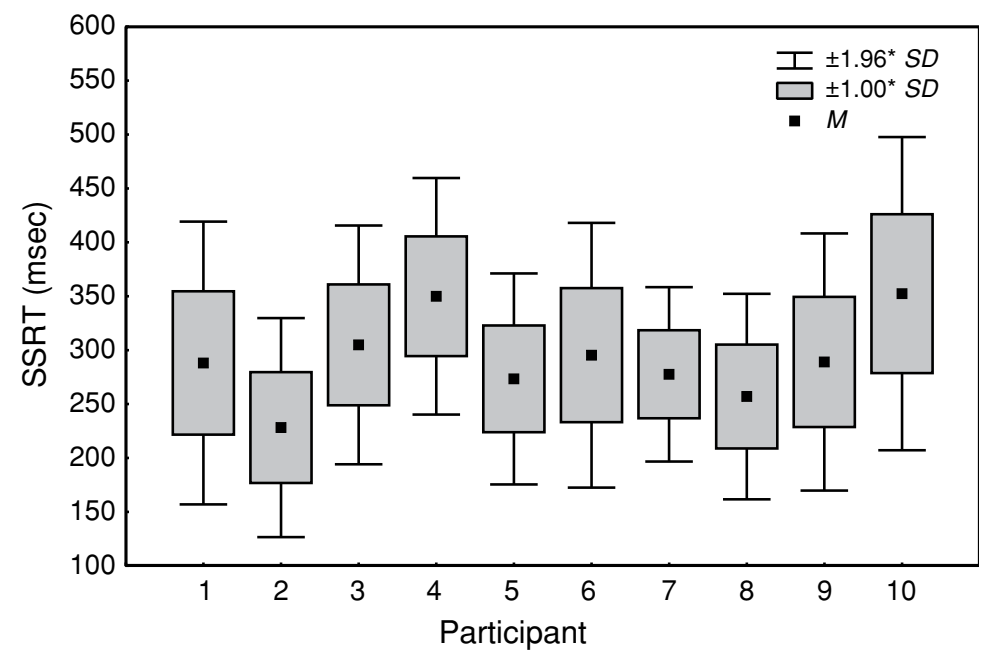

Figure 3. Box- and whiskerplots of the distribution of SSRT for each individual participant in Experiment 1. 
Table 2

Correlations Between the Dependent Measures of SSRT, FINRT, and PE in Experiment 1 for Individual Participants

\begin{tabular}{cccc}
\hline P No. & $\begin{array}{c}\text { SSRT } \\
\text { and PE }\end{array}$ & $\begin{array}{c}\text { SSRT and } \\
\text { FINRT }\end{array}$ & $\begin{array}{c}\text { FINRT } \\
\text { and PE }\end{array}$ \\
\hline 1 & .09 & .92 & .04 \\
2 & .07 & .89 & .09 \\
3 & -.01 & .75 & .06 \\
4 & -.20 & .73 & -.09 \\
5 & .06 & .53 & .30 \\
6 & .11 & .70 & .11 \\
7 & .03 & .68 & .10 \\
8 & -.14 & .61 & -.27 \\
9 & .08 & .50 & .04 \\
10 & .09 & .73 & .14 \\
$M$ correlation & .02 & .74 & .05 \\
\hline
\end{tabular}

Note-P No., participant number; SSRT, stop signal RT; FINRT, final RT; PE, phase error. Mean correlations across participants were calculated by averaging the Fisher's $Z$ transformations of the correlations across participants and then retransforming them.

be computed reliably, or that resulted in values larger than $1,010 \mathrm{msec}$ or smaller than $50 \mathrm{msec}(1.8 \%)$, were subsequently dropped from all analyses. This confirmed the reliability and viability of the analysis algorithm developed on the previous data set. An ANOVA was performed on the RTs, with the factors of response (stop vs. accelerate) and signal (target stop vs. color change). The ANOVA revealed a significant effect of response type $[F(1,11)=28.1, p<$ $.01]$, with mean SSRT being shorter than mean ACCEL RT (358 msec and $449 \mathrm{msec}$, respectively). Likewise, there was a main effect of signal type $[F(1,11)=66, p<.01]$. Mean SSRT/ACCEL RT was shorter when the stimulus was target stopping than when it was color change (364 msec and $442 \mathrm{msec}$, respectively). Finally, there was a marginal interaction $[F(1,11)=4.5, p<.06]$. Planned comparisons indicated that, in keeping with the predictions, target stop led to faster mean SSRT than ACCEL RT (311 msec vs. $418 \mathrm{msec}$, respectively) $[F(1,11)=26.9, p<.01]$. The color change also led to faster mean SSRT than ACCEL RT (405 msec vs. $489 \mathrm{msec}$, respectively), albeit to a lesser degree $[F(1,11)=19.9, p<.01]$. An ANOVA on PE indicated a significant influence for response type $[F(1,11)=6.7, p<$ $.05]$, with tracking being worse for the stop task in comparison with the accelerate task $\left(3.1^{\circ}\right.$ vs. $2.7^{\circ}$, respectively).

The results of Experiment 2 provided converging evidence for the results of Experiment 1. SSRTs were found to be faster than ACCEL RTs, and the target stopping proved to be a more effective stimulus than the color change, albeit to a lesser degree in the accelerate task. The results demonstrate that the dependent measures can be used to address questions about cognitive processes, and they conform to previous response adjustment performance (Pisella et al., 1998). Different signals may prove to be more salient, overall, because target stopping led to faster responses than did color change for both response types. This notion has yet to be explored in the stopping literature. In addition, these results demonstrate that the initiations of different response changes require different latencies, with the initiation of a stop being faster than the initiation of an acceleration.

\section{Conclusions}

The present study introduced and demonstrated the use of a novel tracking task designed to investigate response control measures, in general, and stopping, in particular. The new task offers several advantages in comparison with previous tasks (Logan, 1994; Morein-Zamir \& Meiran, 2003). Unlike the stop signal task, but similarly to the initial tracking task, the present task offers a measurement on every stop trial, with no need to rely on mathematical assumptions such as those used with the racehorse model. The former, in particular, can be significant when patients or groups such as children or the elderly are tested. Measurements on every trial also make additional information available, such as the variability of the stopping distribution, which can be valuable when examining groups suspected of deficient inhibition (see, e.g., Oosterlaan et al., 1998). The new task also offers several advantages in comparison with the initial tracking task, such as more precise measurements, advanced temporal resolution, and a more efficient algorithm for computing SSRT and ACCEL RT. Nevertheless, the task does require specialized hardware to measure response force over time. Likewise, if the tracking task becomes too difficult, performance may become too variable, and the detection of force change would require a more complex algorithm.

Two important differences stand out between the stop signal task and the present tracking task. First, whereas stopping in the former task examines stopping an action before it is initiated, the present task leads participants to stop their action after it has been initiated. Evidence from a study comparing performance in the two tasks supported the conclusion that they are sensitive to the same stopping process (Morein-Zamir et al., 2004). Second, almost all stop signal tasks have utilized a simple buttonpress or some discrete, all-or-none response, whereas the present task examines the unfolding of response force over time. The monitoring of response force, the core of the present task, may prove beneficial even in the use of discrete responses, such as those observed in most of the stopping 
literature (see also van den Wildenberg, van Boxtel, \& van der Molen, 2003).

The two experiments described above demonstrated that numerous task parameters can be examined, including stimulus and response adjustment characteristics. Many additional parameters could be manipulated to alter the task and to address a variety of research questions. For example, although in the present study, the trigger for a signal was dependent on tracking performance, tracking duration can also determine signal onset. Likewise, tracking trials in which no signal is presented can also be introduced, making the task more similar to the traditional stop signal task, in which stop signals are typically present on only a minority of trials (Morein-Zamir et al., 2004). Importantly, the nature of the task can also be altered. For example, Morein-Zamir, Nagelkerke, Chua, Franks, and Kingstone (2006) modified the visual contingencies so that the harder the participant pressed, the slower the response marker moved. In addition, the task need not engage spatial tracking: In order to measure response adjustment, one can envision different visual feedback (e.g., pressing on the sensor to inflate a balloon on the screen).

In conclusion, the use of several converging tasks providing in-depth information on stopping performance could offer much needed insight into the processes governing stopping, in particular, and response control, in general.

\section{REFERENCES}

Band, G. P. H., van der Molen, M. W., \& Logan, G. D. (2003). Horserace model simulations of the stop-signal procedure. Acta Psychologica, 112, 105-142.

De Jong, R., Coles, M. G. H., Logan, G. D., \& Gratton, G. (1990). In search of the point of no return: The control of response processes. Journal of Experimental Psychology: Human Perception \& Performance, 16, 164-182.

Henry, F. M., \& Harrison, J. S. (1961). Refractoriness of a fast movement. Perceptual \& Motor Skills, 13, 351-354.

Hommel, B., \& Prinz, W. (EDs.) (1997). Theoretical issues in stimulusresponse compatibility. Amsterdam: Elsevier

Ladefoged, P., Silverstein, R., \& Papcun, G. (1973). Interruptibility of speech. Journal of the Acoustical Society of America, 54, 1105-1108.

LAPPIN, J. S., \& ERIKSEN, C. W. (1966). Use of a delayed signal to stop a visual reaction-time response. Journal of Experimental Psychology, 72, 805-811.

LoGAN, G. D. (1982). On the ability to inhibit complex movements: A stop-signal study of typewriting. Journal of Experimental Psychology: Human Perception \& Performance, 8, 778-792.

LoGAN, G. D. (1994). On the ability to inhibit thought and action: A users' guide to the stop signal paradigm. In D. Dagenbach \& T. H. Carr (Eds.), Inhibitory processes in attention, memory, and language (pp. 189-239). San Diego: Academic Press.
LogAn, G. D., \& Burkell, J. (1986). Dependence and independence in responding to double stimulation: A comparison of stop, change, and dual-task paradigms. Journal of Experimental Psychology: Human Perception \& Performance, 12, 549-563.

Logan, G. D., \& CowAN, W. B. (1984). On the ability to inhibit thought and action: A theory of an act of control. Psychological Review, 91, 295-327.

Megaw, E. D. (1972). Directional errors and their correction in a discrete tracking task. Ergonomics, 15, 633-643.

Morein-Zamir, S., \& Meiran, N. (2003). Individual stopping times and cognitive control: Converging evidence for the stop signal task from a continuous tracking paradigm. Quarterly Journal of Experimental Psychology, 56A, 469-489.

Morein-Zamir, S., Nagelkerke, P., Chua, R., Franks, I., \& KingSTONE, A. (2004). Inhibiting prepared and ongoing responses: Is there more than one kind of stopping? Psychonomic Bulletin \& Review, 11, 1034-1040.

Morein-Zamir, S., Nagelkerke, P., Chua, R., Franks, I., \& KingSTONE, A. (2006). Compatibility effects in stopping and response initiation in a continuous tracking task. Quarterly Journal of Experimental Psychology, 59, 2148-2161.

Nigg, J. T. (2001). Is ADHD a disinhibitory disorder? Psychological Bulletin, 127, 571-598.

Oosterlaan, J., Logan, G. D., \& Sergeant, J. A. (1998). Response inhibition in $\mathrm{AD} / \mathrm{HD}, \mathrm{CD}$, comorbid $\mathrm{AD} / \mathrm{HD}+\mathrm{CD}$, anxious, and control children: A meta-analysis of studies with the stop task. Journal of Child Psychology \& Psychiatry, 39, 411-425.

Osman, A., Kornblum, S., \& Meyer, D. E. (1986). The point of no return in choice reaction time: Controlled and ballistic stages of response preparation. Journal of Experimental Psychology: Human Perception \& Performance, 12, 243-258.

Pisella, L., Arzi, M., \& Rossetti, Y. (1998). The timing of color and location processing in the motor context. Experimental Brain Research, 121, 270-276.

Proctor, R. W., \& ReEve, T. G. (EDS.) (1990). Stimulus-response compatibility: An integrated perspective. Amsterdam: Elsevier.

Rosenthal, R. (1991). Meta-analytic procedures for social research (Rev. ed.). Newbury Park, CA: Sage.

Slater-Hammel, A. T. (1960). Reliability, accuracy, and refractoriness of a transit reaction. Research Quarterly of the American Association for Health, Physical Education, \& Recreation, 31, 217-228.

TANNOCK, R. (2003). Neuropsychology of attention disorders. In S. J. Segalowitz \& I. Rapin (Eds.), Handbook of neuropsychology (2nd ed., Vol. 8, Part II, pp. 753-784). Amsterdam: Elsevier.

VAN den Wildenberg, W. P. M., van Boxtel, G. J. M., \& VAN DeR Molen, M. W. (2003). The duration of response inhibition in the stopsignal paradigm varies with response force. Acta Psychologica, 114, 115-129.

Vince, M. A., \& Welford, A. T. (1967). Time taken to change the speed of a response. Nature, 213, 532-533.

\section{NOTE}

1. Tracking onset did not prove to be an interesting measure in the two experiments reported, because many anticipations were observed. Additional studies not reported here have been conducted that have demonstrated the reliability of the algorithm at detecting force onset. 
APPENDIX

Software Code (in Pascal 6.0) for Detecting Points of Initial Pressure Onset and Offset

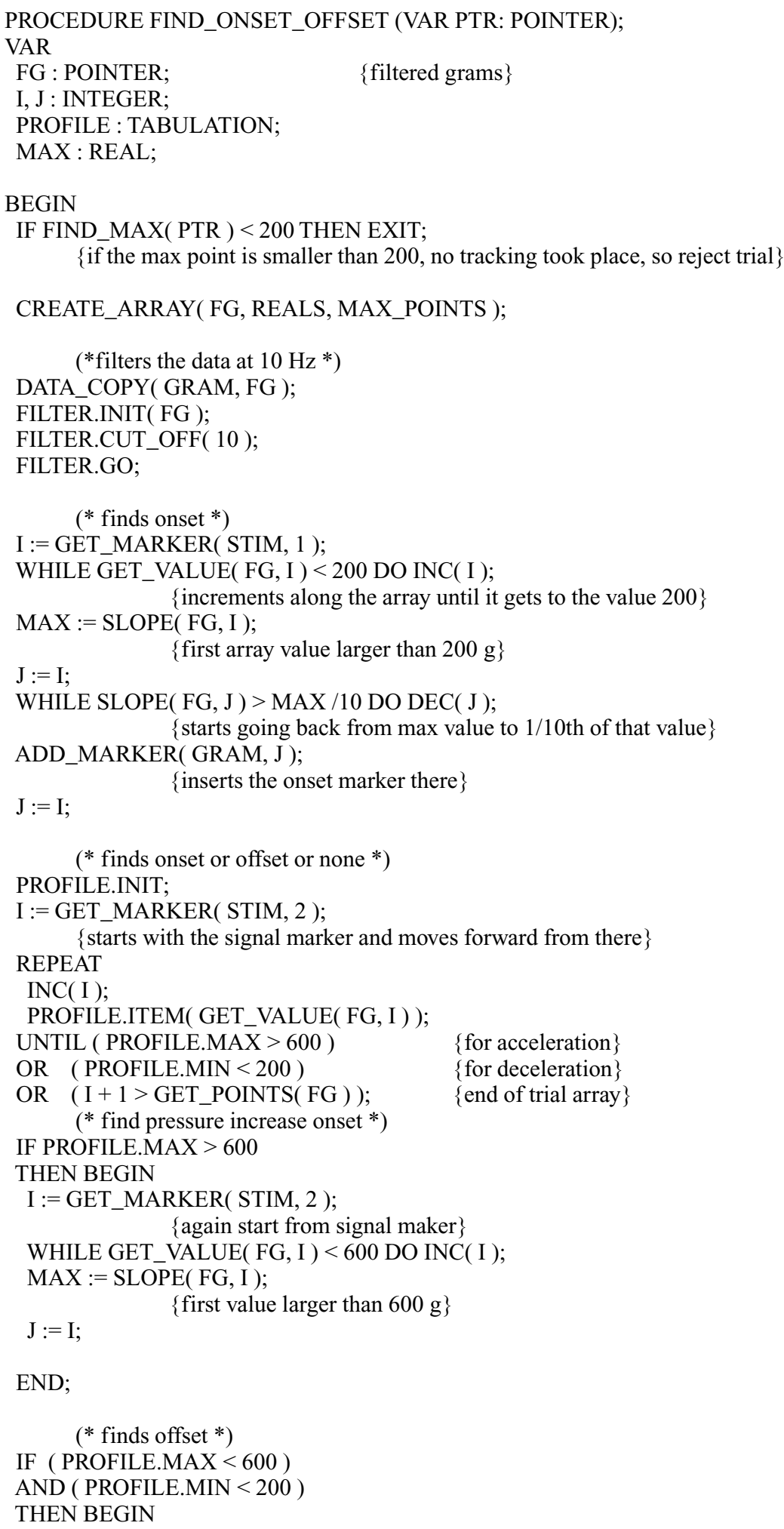

END;

(* finds offset $*$ )

IF ( PROFILE.MAX <600)

AND ( PROFILE.MIN < 200 )

THEN BEGIN 


\section{APPENDIX (Continued)}

I := GET_MARKER( STIM, 2 );

\{again start from signal marker

WHILE GET_VALUE( FG, I ) > 200 DO INC( I );

MAX := SLOPE( FG, I );

$\mathrm{J}:=\mathrm{I}$

\{first value smaller than $200 \mathrm{~g}$ \}

WHILE SLOPE( FG, J ) $<$ MAX /10 DO DEC( J );

ADD_MARKER( GRAM, J );

\{adds a marker at $1 / 10$ th of that value

$\mathrm{J}:=\mathrm{I}$

WHILE SLOPE( FG, J ) $<$ MAX /10 DO INC( J );

ADD_MARKER( GRAM, J );

END;

\{adds a marker at $1 / 10$ th of that value\}

END;

(Manuscript received May 20, 2005;

accepted for publication June 27, 2005.) 